\title{
Prácticas innovadoras de aprendizaje-servicio que promueven el enganche de los menores en la mejora de su comunidad
}

\section{Innovative learning-service practices that get minors hooked on improving their community}

\author{
Domingo Mayor Paredes \\ Universidad de Extremadura, España
}

Autor para correspondencia: domingomp@unex.es

Fecha de recepción: 1 de Julio de 2018 - Fecha de aceptación: 10 de Septiembre de 2018

Resumen: En este trabajo se exponen algunos de los resultados alcanzados en un estudio cualitativo focalizado en el análisis de la influencia que tienen las prácticas de Aprendizaje-Servicio (ApS) para promover la participación fuerte del alumnado de enseñanza primaria en acciones socioeducativas vinculadas a la mejora de su entorno. La investigación tomó como eje de studio un proyecto de ApS que se implementaba en un aula de primaria donde se implicaron: alumnado y profesorado de primaria, estudiantado universitario y agentes socioeducativos comunitarios. Para ello se optó por el estudio de caso como metodología de indagación y el análisis de documentos, la observación participante y las entrevistas semiestructuradas como instrumentos de recogida de información. Los resultados alcanzados permiten constatar los cambios producidos en la participación del alumnado implicado en el proyecto y en el entorno, ya que un espacio físico comunitario que se encontraba abandonado se rehabilitó y se puso en valor a partir del trabajo de los discentes y otros agentes educativos. A partir de los resultados obtenidos se concluye que el ApS abre nuevos horizontes de posibilidad para la articulación de prácticas educativas que contemplan la voz de los menores como un elemento de mejora de la educación escolar.

Palabras clave: aprendizaje-servicio; participación alumnado; mejora escolar; práctica innovadora; comunidad

\begin{abstract}
This paper describes some of the results of a qualitative study focused on analyzing the influence of Learning-Service (LS) practices in promoting strong participation of primary school students in socio-educational action for the improvement of their surroundings. The study axis was a LS project implemented in a primary school classroom which involved primary school students and teachers, university students and community socio-educational agents. The case study was the method of inquiry and document analysis, participant observation and semi-structured interviews were the information collection instruments. The results showed the changes in participation by the students involved in the project and in the setting, which was an abandoned physical community space rehabilitated and revalued by the work of the teachers and other educational agents. These results lead to the conclusion that SL opens new horizons of possibility for the articulation of educational practices including the voices of minors as an element for improving their schooling.
\end{abstract}

Key Words: service-learning; student participation; academic improvement; innovative practice; community 


\section{Introducción}

La conexión entre la escuela o educación formal y la comunidad-ciudad es una idea que ha ido madurando debido a los procesos sociales y educativos que se han ido entrelazando, así como a la identificación y análisis de diferentes prácticas educativas que se estaban implementando fuera del entorno escolar y a la evolución del pensamiento pedagógico (Carbonell, 2014; Dewey, 1950; Freire, 1983; Olsen, 1951; Trilla, 2005, etc.) que pasó de observar y analizar los fenómenos educativos en el ámbito escolar, a significar dichos fenómenos desde una perspectiva holística e integradora.

El reconocimiento progresivo de los espacios múltiples y heterogéneos que iban configurando el nuevo universo educativo ${ }^{1}$ por parte de instituciones internacionales y nacionales y estudiosos de la educación (Asociación Internacional de Ciudades Educadoras, 1990; Carbonell, 2014; Delors, 1996; Trilla, 1993); su formalización disciplinar en áreas de conocimiento como la pedagogía, sociología, psicología, etc.; el potencial educativo de las tecnologías de la información y la comunicación; la necesidad de rentabilizar los recursos públicos y privados insertos en un territorio; la incapacidad de la escuela para hacer frente, por sí sola, a todas las demandas sociales vinculadas a la instrucción y formación integral, etc., son algunos de los elementos que han incidido, por un lado, en la reconfiguración del concepto de educación, ampliando sus contornos más allá del entorno escolar y, por otro, en la búsqueda de nuevas formas de hacer y relacionarse, orientadas a promover sinergias para aprovechar el potencial educativo que encierran las comunidades físicas y virtuales donde la escuela es considerada como un nodo más (Puig et at., 2009; Subirats, 2002) del conjunto de nodos que conforman el tejido de las redes por donde transitan las personas y grupos humanos a lo largo y ancho de su vida.

Siguiendo a Trilla (1995) y Puig et al. (2009) se pueden diferenciar cuatro momentos con diferentes niveles de articulación en el devenir histórico de la institución escolar:

\section{a) La escuela encerrada en sí misma.}

En este primer momento, la escuela tradicional cree tener el saber y los valores necesarios que necesitan los jóvenes. Esta imagen acabada del saber y los valores permite que la escuela se cierre sobre si misma creyendo tener toda la cultura relevante. Este dispositivo no espera nada de la realidad que la circunda.

\section{b) La escuela abierta al medio.}

Este segundo momento representa los postulados de la escuela activa, que considera que el saber y los valores son herramientas que están en continua evolución. Los mismos deben servir para que menores y jóvenes se adapten de forma crítica al medio y aprender a vivir de modo cada vez más feliz y justo. Conciben que la escuela es una preparación para la vida y por ello debe tender puentes que la pongan en relación con la comunidad. En este sentido Olsen (1951, p. 12) planteaba: "La escuela moderna debe aprender a utilizar la comunidad como un laboratorio y libro de texto vivientes para conformar la vida personal y cívica".

\footnotetext{
${ }^{1}$ El universo educativo está conformado por los distintos ámbitos por donde circula la educación: formal, no formal e informal. En él se incluyen los diferentes agentes, instituciones, procedimientos, temáticas, etc. con influencia en el desarrollo individual y social (Trilla, 1993).
} 


\section{c) El medio es educativo.}

Un tercer momento está vinculado al reconocimiento del potencial educativo del territorio sociocultural y económico donde se desenvuelven las personas y grupos humanos. Así lo pusieron de manifiesto los distintos informes internacionales a los que hacíamos referencia anteriormente, dando lugar a la configuración de las tres categorías que conforman el universo educativo actual: educación formal, no formal e informal, para representar las múltiples y heterogéneas prácticas educativas que se estaban implementando al margen del contexto escolar. Desde esta perspectiva, se concibe que el medio como "un libro abierto que puede leerse sensorial y cognitivamente, desde las inteligencias múltiples, y que activa las diversas dimensiones de la educación integral. En definitiva, un espacio de espacios abiertos al aprendizaje y a la socialización" (Carbonell, 2014, p. 32).

\section{d) El medio es una red educativa.}

El cuarto momento, supone un avance cualitativo con relación a la forma de trabajar en el territorio. Las evaluaciones de los procesos puestos en marcha indican la insuficiencia de la escuela y, demás entidades, proyectos o influencias educativas de la sociedad en sí mismas, para dar respuestas eficaces a las nuevas demandas sociales, por lo que hay que avanzar en acciones que se sirvan de todas las redes de aprendizaje existentes en la comunidad (Mayor y Rodríguez, 2015). En esta línea de pensamiento van aflorando prácticas educativas donde la escuela y distintas instituciones públicas y entidades sociales trabajan colaborativamente orientadas por objetivos comunes: programas de Desarrollo Comunitario, Ciudades Educadoras, Planes Educativos de Entorno, Comunidades de Aprendizaje..., y los proyectos de AprendizajeServicio, una práctica educativa sustentada en una doble intencionalidad: pedagógica y social, que ha servido como marco teórico del proyecto objeto de indagación que analizamos en este estudio.

\section{Aprendizaje-Servicio: una práctica educativa de naturaleza compleja}

Indagando en los albores del siglo XX podemos identificar a diferentes autores, postulados teóricos y prácticas que configuran las distintas líneas de intersección o itinerarios que dibujan este universo en construcción. Diversos estudiosos (Máximo, 2010; Puig y Palos, 2006) plantean que los primeros antecedentes de esta metodología podemos encontrarlos en el surgimiento, a principios de siglo XX, de la corriente educativa liderada, entro otros, por Dewey y James. Dewey defendía el aprender haciendo, y James propuso el servicio civil a la comunidad en sustitución del servicio militar, dando lugar a las primeras experiencias estructuradas de servicio comunitario estudiantil.

Asimismo, las ideas del pedagogo brasileño Paolo Freire, expuestas en su obra pedagogía del oprimido (1983), influyeron significativamente en los pioneros del ApS, tanto en EE. UU. donde estuvo exiliado algunos años-, como en América Latina.

Por otro lado, el ApS, en su devenir sociohistórico, ha sido interpretado y materializado de múltiples formas como lo evidencian las diversas definiciones y prácticas halladas. Un estudio realizado por Kendal (1990 citado en Martínez-Odría, 2008, p. 629) encontró hasta 147 nociones diferentes en la literatura especializada para significar esta práctica educativa de naturaleza multidimensional (instituciones y agentes que intervienen, ámbitos donde se desarrolla, 
dimensiones pedagógicas que la constituyen, tipología de servicios, edades de los participantes, etc.) y multidisciplinar (pedagogía, psicología, sociología, etc.) que se viene implementando por múltiples agencias en diversos contextos socioculturales.

A pesar de las diferencias y similitudes que guarda con otras prácticas experienciales (trabajo de campo, iniciativas solidarias y servicios comunitarios), el estudio científico de la evolución de dicho fenómeno ha posibilitado "un consenso a nivel internacional que apunta a definir al aprendizaje-servicio a partir de tres ejes fundamentales" (Montes, Tapia y Yaber, 2011, p. 9):

- Se trata de un servicio a la comunidad con el propósito de dar una respuesta a necesidades reales y sentidas.

- Existe una planificación intencional e integrada de los contenidos curriculares y las actividades relacionadas que conforman el servicio a la comunidad.

- El protagonismo fuerte del alumnado durante el diseño, implementación y evaluación de las acciones educativas.

En el ámbito iberoamericano se suele asumir la definición de ApS planteada por Puig, Palos (2006, p. 4): "Es una propuesta educativa que combina procesos de aprendizaje y de servicio a la comunidad en un solo proyecto bien articulado en el que los participantes se forman al trabajar sobre necesidades reales del entorno con el objetivo de mejorarlo".

Las prácticas de ApS se constituyen como actividades complejas que quiebran la forma de enseñanza de los dispositivos escolares tradicionales, basada en aprendizajes declarativos abstractos, descontextualizados y de escasa relevancia social (Pérez, 2012). Esta forma de enseñar se traduce en aprendizajes poco significativos, relevantes y útiles, y en la incapacidad del alumnado para transferir y generalizar lo que aprenden a situaciones de la vida real. En cambio, en las acciones de ApS los aprendizajes, al estar imbricados con el servicio, favorecen la comprensión problemática de la realidad donde se va a actuar: diagnosticar y analizar las necesidades sociales a las que se pretende dar respuesta; implementar un servicio adecuado a dichas necesidades y reflexionar sobre lo acontecido. Todo ello favorece la significatividad, relevancia y utilidad de los aprendizajes, ya que son puestos en acción para clarificar y afrontar problemas básicos de la comunidad y para ampliar conocimientos, sensibilidades y afectos.

\section{La participación fuerte de los menores en las prácticas de Aprendizaje-Servicio}

En el universo educativo, la participación puede interpretarse de distintos modos: como derecho, objetivo, proceso, medio, contexto, etc. (Llena, Parcerisa y Úcar, 2009), significando con ello la diversidad de finalidades que persiguen las acciones que se implementan en los ámbitos de la educación formal y no formal.

En el ámbito de la educación escolar, se viene utilizando, en general, como un mecanismo burocrático al servicio de la gestión. En este sentido, Feíto (2011, p.112) plantea que "todos los estudios que han analizado la participación del alumnado refieren una situación que oscila entre la sumisión y la indiferencia”. 
Asimismo, se emplea, en el ámbito del aula, para describir situaciones que conllevan desiguales niveles de implicación por parte del alumnado: escuchar, opinar, gestionar algo, estar apuntado a, o ser miembro de, tomar decisiones, etc. Ello pone de relieve la existencia de múltiples formas, tipos, grados y ámbitos de participación (Trilla y Novella, 2011).

Para clarificar dichos asuntos, han desarrollado variados análisis (Fielding, 2011; Hart, 1993; Trilla y Novella, 2001) que señalan las características de los diversos tipos de participación y los diferentes grados de implicación que conllevan. En este trabajo seguiremos la propuesta planteada por Puig et al. (2017) y Rubio et al. (2015).

Tabla 1. Distintos tipos de participación de los y las menores

$\begin{array}{llll}\text { Cerrada } & \text { Delimitada } & \text { Compartida } & \text { Liderada } \\ \text { Los participantes se limitan a } & \begin{array}{l}\text { Los participantes } \\ \text { realizar las tareas que }\end{array} & \begin{array}{l}\text { Los participantes } \\ \text { comparten con sus }\end{array} & \begin{array}{l}\text { Los participantes se } \\ \text { convierten en promotores }\end{array} \\ \text { previamente se han } & \text { puntuales requeridas } & \text { educadores la } & \text { y responsables del } \\ \text { programado para el desarrollo } & \text { por los educadores en } & \text { responsabilidad en el } & \text { proyecto de modo que } \\ \text { de la actividad, sin la } & \text { distintos momentos } & \text { diseño y desarrollo del } & \text { intervienen en todas sus } \\ \text { posibilidad de introducir } & \text { del proceso. } & \text { conjunto de la actividad. } & \text { fases, decidiendo sobre } \\ \text { modificaciones a la propuesta } & & & \text { aspectos relevantes. } \\ \text { inicial. } & & & \end{array}$

Fuentes: Puig, et al., 2017 y Rubio et al., 2015.

Del modelo de participación expuesto anteriormente, conviene señalar algunas cuestiones:

a) Los diversos tipos de participación reflejan, por un lado, maneras de participar cualitativa y cuantitativamente distintas ya que favorecen desiguales niveles de implicación, así como la puesta en acción de distintas capacidades cognitivas, afectivas, comportamentales y relacionales. Por otro lado, evidencian la concepción de educación, alumnado o participantes y de práctica educativa que tiene la institución o personas encargadas de llevar a cabo la acción.

b) Las formas de participación no son excluyentes, ya que la participación cerrada o débil, puede ser necesaria, en un primer momento, para el progreso hacia formas de participación más complejas, fuertes o compartidas. En este sentido, es importante identificar cómo y para qué se ponen en marcha los procesos participativos y el grado de protagonismo que asumen los participantes, ya que puede ser un elemento que sustenta modelos de educación cívica radicalmente diferentes (Susinos y Ceballos, 2012).

c) Las prácticas educativas orientadas a favorecer la participación fuerte o negociada de los participantes conciben a la misma como un objetivo y proceso de aprendizaje. Como plantean Llena, Parcerisa y Úcar $(2009$, p. 124) ser parte o tomar parte de una acción educativa es "un proceso en forma de bucle continuo a través del cual las personas aprendemos a participar participando. Y, participando, aprendemos a ser personas y miembros de una comunidad concreta".

Con relación a la participación en los proyectos de $\mathrm{ApS}$, se piensa desde una dimensión fuerte y negociada, debido a que es una de sus señas de identidad junto a la vinculación de los aprendizajes y el servicio a la comunidad con el propósito de mejorarla, a partir de la cual se configuran los mismos. En este sentido, el ApS se postula como una pedagogía de lo 
significativo y de lo que abre nuevas posibilidades (Puig y Palos, 2006), donde los participantes se involucran en asuntos públicos, asumiendo la responsabilidad que de ellos se derivan en las distintas etapas del proyecto: desde la elección del tema sobre el que se trabajará, hasta la implementación de las acciones planificadas y la evaluación de la experiencia.

Además, la participación es planteada como un proceso de aprendizajes múltiples y multidireccionales, ya que las prácticas de $\mathrm{ApS}$, se articulan como un escenario complejo donde confluyen distintos elementos (grupo de iguales, relaciones con personas de la comunidad, actividades en el medio real, aprendizajes orientados a la acción, reflexión de las experiencias, etc.). Todo ello favorece que los protagonistas de la experiencia vayan desarrollando sus capacidades cognitivas, afectivas, comportamentales y relacionales.

\section{Metodología}

\section{Escenario de la investigación}

La investigación se llevó a cabo en un centro educativo público, ubicado en un barrio socialmente desfavorecido, alejado del centro de la ciudad, y catalogado por las administraciones públicas como Zona con Necesidades de Transformación Social, debido a las situaciones de exclusión social que vive una parte significativa de la población: altos índices de desempleo, escasos niveles de formación para acceder a los nuevos yacimientos de empleo, altas tasas de absentismo escolar en primaria y abandono escolar en secundaria, etc. (Junta de Andalucía, 2005).

En dicho contexto se vienen implementado un programa de políticas públicas en materia de vivienda que se materializaron en el Plan de Reforma del Barrio. Con el mismo se pretende mejorar la calidad de vida de la población. Para ello se han diseñado distintas actuaciones urbanísticas que van acompañadas de un trabajo socioeducativo con la población. Una de esas acciones socioeducativas está configurada por el Programa Pedagogía del Hábitat ${ }^{2}$ que tiene, entre otros objetivos, promover sinergias entre las distintas instituciones y profesionales que trabajan en la zona: educación, vivienda, salud, entidades sociales, universidad de Almería, etc.).

\section{Proyecto objeto de estudio}

Una de esas acciones coordinadas puestas en marcha fue el proyecto socioeducativo para la rehabilitación del Parque Garlochi ${ }^{3}$. Un espacio público abandonado que se había convertido en un vertedero de basura.

Para la rehabilitación del mismo se contó con la colaboración de distintos agentes e instituciones y se estructuró un proyecto de ApS que tenía como propósitos: Promover sinergias educativas entre la escuela-comunidad-universidad; favorecer la construcción de una ciudadanía activa, responsable y crítica y desarrollar metodologías innovadoras que promuevan aprendizajes significativos y útiles, dirigidos a la mejora de los espacios públicos.

\footnotetext{
${ }^{2}$ http://www.elalmeria.es/almeria/pequenos-Chanca-Puche-comodos-habitables_0_318868493.html\#!

${ }^{3}$ Para más información sobre el proyecto: https://www.youtube.com/watch?v=Hyfb1FU8WcQ\&t=960s
} 


\section{Enfoque metodológico}

En coherencia con el propósito del estudio, se optó por un enfoque metodológico de naturaleza cualitativa, ya que favorece la comprensión de los fenómenos sociales desde la perspectiva de sus actores y, además, aporta una mirada de los problemas educativos más amplia, rica y completa, pues toma en cuenta el contexto donde se producen los hechos (Taylor y Bogdan, 1992). Se utilizó el estudio de caso, con el propósito de comprender y explicar la singularidad y complejidad de un fenómeno singular (Simons, 2011): la influencia de un proyecto de ApS en la participación fuerte del alumnado orientada a mejorar su entorno.

\section{Informantes clave}

Las personas que participaron, previo consentimiento informado, fueron: 9 profesores y 16 alumnos de primaria que ejercían como representantes de alumnos de 8 aulas comprendidas entre $3^{\circ}$ y $6^{\circ}, 6$ alumnos y 1 profesora de la universidad del grado de Educación Social y 2 representantes de entidades sociales. Para su selección se siguieron los siguientes criterios: obtener la máxima rentabilidad de aquello que aprendemos y la disponibilidad e interés de las personas implicadas para colaborar positivamente (Stake, 2010).

\section{Procedimiento e instrumentos de recogida de información}

El proceso de indagación se realizó a lo largo de un curso escolar, contando con la presencia del investigador en el aula, y otros lugares del barrio, dos días a la semana durante cuatro horas diarias, con el propósito de rastrear y obtener los datos en el contexto concreto, para la cual utilizamos diversos métodos cualitativos (Taylor y Bogdan, 1992):

- El Análisis de documentos se centró en el Plan de Rehabilitación del Barrio, Proyecto Educativo de Centro, Programa de la asignatura de Innovación Educativa, Diario de prácticas del alumnado de la Universidad y distintos materiales didácticos elaborados por el alumnado de primaria.

- La observación participante se llevó a cabo en el aula de educación primaria y en distintos espacios del barrio utilizados por el alumnado durante el trabajo en el Parque Garlochí.

- La entrevista semiestructurada en profundidad individual fue dirigida a maestras y maestros y alumnado de primaria, profesora y alumnado de la Facultad de CC. de la Educación de la Universidad de Almería y representantes de entidades sociales del barrio.

- La entrevista semiestructurada grupal fue destinada a alumnado de primaria y de la universidad.

Los datos obtenidos en el curso de la acción fueron analizados siguiendo la técnica de análisis de contenido, con el interés de configurar las categorías o asuntos más relevantes. Para ello se llevó a cabo un conjunto de tareas de comparación, contrastación, agregación y ordenación sobre los datos, que permitieron identificar las unidades de significado, codificar las mismas y posteriormente conformar las temáticas que se presentarán en una tabla posteriormente.

Con el propósito de intentar asegurar la credibilidad y replicabilidad de los datos (Pérez, Galán y Quintanal, 2012), favorecer la triangulación entre instrumentos y eliminar el sesgo del 
investigador, las observaciones y las entrevistas realizadas fueron grabadas en vídeo y, posteriormente, se realizó la transcripción literal de todo el material recogido, presentándose la misma a los informantes para obtener su validación.

Por tanto, la información expuesta en el siguiente epígrafe es el resultado de contrastar permanentemente los asuntos de interés que se iban obteniendo a través de los diferentes instrumentos de recogida de datos.

\section{Resultados}

Los resultados de tipo cualitativo se expresan de manera narrativa, sistematizados en función del grupo de actores que toma la palabra y con descripciones de algunos de los asuntos más significativos que fueron aflorando durante la investigación.

\section{Menores enganchados en un proceso educativo al que pueden dotar de sentido y sentirse reconocidos}

Después de las primeras sesiones formativas realizadas en el aula de música del centro escolar, los representantes de alumnos de $3^{\circ}, 4^{\circ}, 5^{\circ}$ y $6^{\circ}$ informaron a sus compañeros de aula de las actividades realizadas y de su participación en la rehabilitación del Parque Garlochí, ello motivó que muchos menores quisieran participar en el proyecto.

Durante el recorrido por el centro, hasta llegar al aula donde se desarrollaba la actividad, el Educador Social, coordinador del proyecto, recibía múltiples peticiones de discentes: ¿puedo participar yo en el arreglo del parque?, era una pregunta que se repetía continuamente durante la estancia en el colegio $\left(\mathrm{D} . \mathrm{O}^{4}\right)$.

El interés despertado en los menores del colegio (Tabla 2) provocó que la Jefa de Estudios, responsable de la planificación de las salidas al barrio, se replanteara el proyecto inicial, modificándose el número de salidas y ampliándose los grupos que participarían en distintas actividades relacionadas con la rehabilitación del parque (limpieza, pintura, entrevistas, decoración, etc.).

El interés inicial de algunos de ellos estaba centrado en la realización de una actividad ubicada fuera de la rutina marcada por el currículo del centro. Muchos de los menores planteaban: "el colegio es aburrido; se habla de muchas cosas que yo no sé, ni me interesan, etc. Posteriormente, a medida que avanzaba el proceso y se abrían espacios de reflexión sobre la práctica, fueron aflorando intereses relacionados con el compromiso, la solidaridad, el cuidado y la diversión.

\section{Tabla 2. ¿Qué plantea el alumnado que ha participado en el proyecto?}

\begin{tabular}{ll}
\hline Asuntos que destacan & $\mathrm{N}^{\mathrm{o}}$ de alumnos/as \\
\hline
\end{tabular}

\footnotetext{
${ }^{4}$ Abreviaturas utilizadas en el trabajo: Diario de Observación (D.O.); Entrevista Profesorado Primaria (Ent. P. P.); Entrevista Alumnado Primaria (Ent. A. P.) y Entrevista Alumnado Universitario (Ent. A. U.).
} 
1. Autoevaluación del interés:

-Interés alto en participar.

2. Razones de la participación:

-Para construir un sitio donde los niños puedan jugar. 14

-Para tener un barrio limpio y saludable. 10

-Porque el colegio es aburrido. 8

3. Aprendizajes adquiridos:

-Trabajar en equipo.

-A elaborar mezcla, pintar, hacer entrevistas, etc.

-Compartir.

-Conocimiento mayor del barrio.

4. Pedido a los vecinos:

-Que lo cuiden (que no lo rompan, que no tiren basura al suelo, etc.) 16

5. Pedido al Ayuntamiento:

-Que ponga papeleras y que lo limpie.

-Que ponga vigilancia.

6. Sentimientos despertados:

-Contento. Por qué he participado en el arreglo del parque.

-Feliz. Por qué hemos trabajado muy bien juntos. 10

7. Palabra o frase que simboliza la experiencia:

-Esfuerzo.

-Satisfacción por el arreglo.

-Participación.

-Diversión.
(14)

(10)

10

8

6

11

5

13

7

6

6

Fuente: Elaboración propia.

Otro asunto relevante fue el deseo de los y las menores de realizar entrevistas a distintos profesionales del barrio y al vecindario. Esta actividad, que ellos denominaban como "hacer de periodistas" (Ent. A.P. 2), le brindaba la posibilidad de reconectar con la lecto-escritura comprensiva, ya que tenían que recoger las opiniones de personas conocidas sobre un asunto que ellos consideraban importante: "la construcción de su parque" (Ent. A. P. 4) y, posteriormente, analizar las respuestas. Asimismo, era una oportunidad de sentirse reconocidos y valorados por personas significativas del barrio: "He entrevistado a la enfermera y a mi médica y les ha gustado mucho lo que estamos haciendo", le comentaba efusivamente una menor a un grupo de compañeras (D.O.).

A pesar del esfuerzo físico realizado durante la rehabilitación del Parque: limpieza del espacio, hacer mezcla, pintar y decorar las paredes y realización de entrevistas y fotografías, "los y las menores preferían salir a trabajar antes que participar en las actividades curriculares" (Ent. P. P.1). En este sentido la Jefa de Estudios del colegio planteaba: "Tenemos que empezar a pensar como mejoramos los procesos de enseñanza-aprendizaje para que los menores vengan contentos a la escuela. Con este proyecto hemos aprendido mucho".

En este orden de ideas, el profesorado entrevistado (9), que participó durante la fase de implementación del proyecto, planteaba como fortalezas del proyecto las siguientes: " $\mathrm{Ha}$ despertado el deseo de aprender y la asistencia regular del alumnado al centro"; "ha promovido la implicación de los menores en el conjunto de las actividades"; "se ha producido una alta implicación del profesorado", así como "la utilidad social y educativa del proyecto". 
Asimismo, el alumnado universitario, que al principio era reticente a participar en el proyecto por la imagen mental que se había ido construyendo en base a algunas noticias sensacionalistas aparecidas en algunos medios de comunicación, ya que nunca habían visitado el barrio, exponía: "A través de estos proyectos los menores aprenden a trabajar en grupo, valores y a cuidar y participar en su entorno" (Ent. A. U. 5); "Me ha sorprendido positivamente el interés mostrado por los niños y su gran implicación en las actividades" (Ent. A. U. 2).

Durante el desarrollo de la experiencia, los menores aprendieron a organizarse en pequeños grupos para llevar a cabo las distintas tareas que requería el proyecto propuesto por ellos. Cuando se les pregunta por los aprendizajes adquiridos, las respuestas que más se repetían estaban relacionadas con el trabajo en equipo, la elaboración la mezcla y pintura, la realización de fotografías y vídeos, compartir el trabajo con otros compañeros y un mayor conocimiento del barrio. Este último asunto fue analizado con el alumnado en varias sesiones formativas (D.O.). Durante las mismas estuvieron investigando en la corresponsabilidad de distintos agentes (vecinos, administración pública y profesionales) y en el uso y cuidado de los espacios públicos. Posteriormente, los menores decidieron realizar una campaña de sensibilización que consistía en el diseño y pegada de carteles en distintos lugares del barrio, en una carta dirigida al Alcalde y una nota de prensa ${ }^{5}$ para hacer visible su trabajo (D.O.).

Generar espacios y tiempos para que las voces de los menores se reconozcan y valoren posibilitó un nivel elevado de compromiso y de satisfacción del alumnado en las acciones implementadas. En este sentido, cuando se le preguntaba a los mismos exponían: "Me he sentido muy bien, porque hemos trabajado en un proyecto diseñado por nosotros que va a servir para que los pequeños puedan jugar" (Ent. A. P. 6). "Me siento feliz porque hemos trabajado muy bien juntos en algo que nos gustaba" (Ent. A. P. 10).

Interés, compromiso y satisfacción de los menores fueron algunas de los asuntos que señalaba el profesorado de primaria que acompañaba en las salidas. A partir de estas observaciones planteaba como propuestas de mejora, para el próximo proyecto, las siguientes: "Incluir el proyecto en el proyecto curricular de centro" (Ent. P. P. 2); "desarrollar el proyecto durante más tiempo" (Ent. P. P. 6) y "pensar en cómo incorporar a las familias" (Ent. P. P. 3). Propuestas que se fueron construyendo a partir del establecimiento de relaciones colaborativas entre diversos agentes educativos provenientes del campo de la educación formal y la educación social y de participar en acciones formativas que utilizaban otras metodologías y otros sentidos de la educación. Ello suponía abrir una brecha para repensar las prácticas escolares que, hasta ese momento, estaban instauradas en el centro (D.O.).

\section{Conclusiones y discusión}

A luz de los resultados logrados en este estudio podemos destacar dos dimensiones nucleares que influyeron notablemente en la participación de los menores: las actividades conectadas a sus intereses ("La elección del servicio la hizo el alumnado" -Ent. P. P. 3- ) y los

\footnotetext{
${ }^{5}$ http://www.noticiasdealmeria.com/noticia/76901/capital/ninos-y-jovenes-de-el-puche-rehabilitan-el-parquegarlochi-tras-un-proceso-participativo-con-las-familias-del-barrio-.html
} 
diferentes niveles de implicación de los mismos en las acciones que comprendían cada una de las fases del proyecto. Estos dos elementos, en la práctica, se hayan imbricados retroalimentándose mutuamente. Tomando en consideración dichas dimensiones queremos destacar algunas cuestiones.

En primer lugar, a lo largo del proceso de observación pudimos detectar que la materialización de la participación del alumnado se va configurando en un escenario dinámico y relacional donde convergen, además de las dimensiones señaladas anteriormente, los elementos estructurales de los proyectos de ApS. En esta línea, las prácticas de ApS, como ya hemos señalado, se van conformando a partir de tres ejes, donde la partición activa del alumnado en las distintas fases del proyecto es considerada un elemento nuclear de las mismas (Díaz, 2006; Furco, 2007; Puig et al., 2009; Tapia, 2001). Aunque este marco referencial puede ser interpretado de forma diferente por distintos agentes, ofrece un escenario de posibilidad para desarrollar las capacidades de los menores como actores sociales, favoreciendo así la participación negociada o auténtica (Hart, 1993; Rubio et al., 2015) que conlleva un protagonismo fuerte del alumnado en el acontecer de la experiencia educativa.

En este estudio hemos observado que, para conseguir ese nivel de implicación fuerte, la participación de los infantes tiene que ser considerada como un objetivo educativo orientado a favorecer la adquisición progresiva de capacidades complejas o de orden superior: Planificación de las acciones, resolución de problemas, tomas de decisiones, aprender a aprender, transferencia de los aprendizajes, etc. (Pérez, 2012) que conllevan un nivel alto de implicación y la circulación por diferentes tipos de participación (cerrada, delimitada, compartida), que en muchas actividades se hallaban conectadas. Esta conclusión es coincidente, en algunos aspectos, con las investigaciones recogidas y efectuadas por UNICEF que evidencian que, "con un adecuado acompañamiento adulto según el caso, los niños y niñas son capaces de planificar, gestionar y realizar cambios importantes en su medio ambiente" (Casas, et at., 2008, p. 30).

Finalmente, se ha constatado las bondades pedagógicas de la participación negociada. Un proceso que implica configurar las prácticas desde una relación más horizontal y acompañar los procesos de creación y adquisición de aprendizajes de los menores generando espacios para que su voz sea escuchada en los procesos de toma de decisiones.

Ello coincide con un variado corpus de investigaciones que señalan los beneficios de la participación fuete del alumnado en las prácticas de ApS, ya que favorece la adquisición de aprendizajes significativos y útiles orientados a conseguir mejoras en el ámbito escolar y social (Fielding, 2011; Mayor y Rodríguez, 2015; Susinos y Ceballos, 2012), así como el desarrollo de su autoestima (Furco, 2007) y la satisfacción personal (Folgueiras, Luna y Puig, 2014).

En relación con la prospectiva, se propone avanzar en distintas líneas:

- Indagar las razones que sustentan la participación de los distintos agentes implicados en las prácticas de ApS.

- Explorar la influencia de esta experiencia en la formación del alumnado de primaria y de la universidad a medio-largo plazo. 
- Analizar la influencia de los proyectos de ApS en los cambios y mejoras producidas en el centro educativo y en la comunidad.

\section{Bibliografía}

Asociación Internacional de Ciudades Educadoras (1990). Carta de ciudades educadoras. Recuperado de http://www.edcities.org/carta-de-ciudades-educadoras/

Casas, F., González, M., Carme, M., Navarro, D., Malo, S., Figuer, C. y Bertran, I. (2008). Informe sobre experiencias de participación social efectiva de niños, niñas y adolescentes. Madrid: Ministerio de Educación, Política Social y Deporte.

Carbonell, J. (2014). Pedagogías del S. XXI. Alternativas para la innovación educativa. Barcelona: Octaedro.

Delors, J. (coord.). (1996). La educación encierra un tesoro. Madrid: Santillana.

Dewey, J. (1950). Las escuelas de mañana. Buenos Aires: Losada.

Díaz, F. (2006). Enseñanza situada: vínculos entre la escuela y la vida. México: McGraw-Hill Interamericana.

Feito, R. (2011). Los retos de la participación escolar: elección, control y gestión de los centros educativos. Madrid: Morata.

Fielding, M. (2011). La voz del alumnado y la inclusión educativa: una aproximación democrática radical para el aprendizaje intergeneracional. Revista Interuniversitaria de Formación del Profesorado, 70(25,1), 31-61. Recuperado de http://www.redalyc.org/pdf/274/27419147003.pdf

Folgueiras, P., Luna, E. y Puig, G. (2014). El Aprendizaje y servicio en educación secundaria. Revista Iberoamericana de Educación, 64(2), pp.1-15. Recuperado de http://www.rieoei.org/deloslectores/5994Luna.pdf

Freire, P. (1983). La educación como práctica de la libertad. España: Siglo XXI.

Furco, A. (2007). Impactos de los proyectos de aprendizaje-servicio. En A. González (coord.). Antología 1997-2007. Seminarios Internacionales de Aprendizaje y Servicio Solidario, pp. 175-183. Buenos Aires: EUDEBA. Editorial Universitaria de Buenos Aires.

Hart, R. (1993). La participación de los niños. De la participación simbólica a la participación auténtica. Ensayos innocenti, 4, 1-48. Santafé de Bogotá. UNICEF. Recuperado de http://www.unicef-irc.org/publications/538

Junta de Andalucía (2005). Actuaciones en Barriadas y Zonas con Necesidades de Transformación Social. Consejería de Igualdad y Bienestar Social. Recuperado de 
http://www.juntadeandalucia.es/igualdadybienestarsocialopencms/system/bodies/Zonas_ Transformacion_Social/Publicacion/memoria_2005/Memoria_Barriadas_2005.pdf

Llena, A., Parcerisa, A. y Úcar, X. (2009). 10 ideas claves. La acción comunitaria. Barcelona: Graó.

Martínez-Odria, A. (2008). Service-learning o aprendizaje-servicio. La apertura de la escuela a la comunidad local como propuesta de educación para la ciudadanía. Bordón, 59(4), pp. 627640. Recuperado de http://redined.mecd.gob.es/xmlui/handle/11162/36482

Mayor, D. y Rodríguez, D. (2015). Aprendizaje-Servicio: construyendo espacios de intersección entre la escuela-comunidad-universidad. Profesorado. Revista de currículum y formación del profesorado, 19(1), pp. 262-279. Recuperado de http://www.ugr.es/ recfpro/rev191ART11.pdf

Máximo, E. (2010). Aprendizaje-Servicio en América Latina: apuntes sobre pasado y presente. Revista Científica Tzhoecoen, 5, pp. 108-125. Recuperado de http://www.clayss.org.ar/archivos/TZHOECOEN-5.pdf

Montes, R., Tapia, M., y Yaber, L. (2011). Manual para docentes y estudiantes solidarios. Buenos Aires: CLAYSS. Recuperado de http://www.clayss.org.ar/04_publicaciones/Natura2013.pdf

Olsen, E. (1951). La escuela y la comunidad. México: Unión Tipográfica Editorial HispanoAmericana.

Pérez, A. I. (2012). Educarse en la era digital. Madrid. Morata.

Pérez, R., Galán, A. y Quintanal, J. (2012). Métodos y diseños de investigación en educación. España: UNED-Universidad Nacional de Educación a Distancia.

Puig, J. M. (coord.). (2009). Aprendizaje Servicio. Educación y compromiso cívico. Barcelona: Graó.

Puig, J. M. y Palos, J. (2006). Rasgos pedagógicos del aprendizaje-servicio. Cuadernos de Pedagogía, 357, pp. 60-63.

Puig, J. M., Martín, X. y Rubio, L. (2017). ¿Cómo evaluar proyectos de aprendizaje servicio? Voces de la Educación, 2(2), pp. 122-132. Recuperado de http://www.revista.vocesdelaeducacion.com.mx/index.php/voces/article/view/72/63

Rubio, L., Puig, J. M., Martín, X. y Palos, J. (2015). Analizar, repensar y mejorar los proyectos: una rúbrica para la autoevaluación de experiencias de aprendizaje servicio. Profesorado. Revista de currículum y formación del profesorado, 1(19), pp. 111-126. Recuperado de http://www.ugr.es/ recfpro/rev191ART6.pdf 
Simons, H. (2011). El estudio de caso: Teoría y práctica. Madrid: Morata.

Subirats, J. (2002). El factor proximidad y de comunidad en las políticas educativas. En J. Subirats (coord.). Gobierno Local y Educación. La importancia del territorio y la comunidad en el papel de la escuela, pp. 21-50. Barcelona: Ariel.

Susinos, T. y Ceballos, N. (2012). Voz del alumnado y presencia participativa en la vida escolar. Apuntes para una cartografía de la voz del alumnado en la mejora educativa. Revista de Educación, 359, 24-44._DOI: 10.4438/1988-592X-RE-2012-359-194

Stake, R.E. (2010). Investigación con estudio de casos. Madrid: Morata.

Tapia, Ma . N. (2001). La solidaridad como pedagogía. El aprendizaje-servicio en la escuela. Buenos Aires: Ciudad Nueva.

Taylor, S. J. y Bogdan, R. (1992). Introducción a los métodos cualitativos de investigación. Barcelona: Paidós.

Trilla, J. (1993). La educación fuera de la escuela. Barcelona: Ariel.

Trilla, J. (2005). La idea de ciudad educadora y escuela. Revista Educación y Ciudad, 7, pp. 73106. Recuperado de https://dialnet.unirioja.es/servlet/articulo?codigo=5705054

Trilla, J. y Novella, A. M. (2011). Educación y participación social de la infancia. Revista Iberoamericana de Educación, 26, pp. 137-164. Recuperado de http://diposit.ub.edu/dspace/bitstream/2445/56924/1/502327.pdf 